\title{
Two-user Cooperative Transmission Using Superposition Modulation and Soft Information Combining
}

\author{
Rui Lin, Philippa A. Martin and Desmond P. Taylor \\ Department of Electrical and Computer Engineering \\ University of Canterbury \\ Christchurch, New Zealand \\ E-mail:rli24@student.canterbury.ac.nz,p.martin@elec.canterbury.ac.nz, taylor@elec.canterbury.ac.nz
}

\begin{abstract}
We propose a cooperative transmission scheme for two users with one common relay using superposition modulation. It uses distributed Turbo codes (DTCs) for each user. The relay always decodes, then interleaves and re-encodes the decoded data. A new packet is formed by combining the re-encoded packets from both users using superposition modulation. This packet is forwarded to the destination with the average receive SNRs of each user. The destination uses the cooperative Turbo decoder of [1] to improve performance. Simulation results show the proposed scheme outperforms XOR based schemes and is simple to implement.
\end{abstract}

\section{INTRODUCTION}

Cooperative communications has been shown to provide diversity gains in systems with limited numbers of transmit antennas through the use of relay nodes. Several protocols have been proposed to achieve this including amplify-andforward (AF) and decode-and-forward (DF) [2]. However, the gain is achieved at a cost of throughput because transmission is normally divided into the two stages of broadcasting and relaying. This presupposes wireless nodes cannot transmit and receive in the same channel (half duplex).

In order to recover some of the throughput loss, numerous schemes have been proposed $^{1}[3]-[11]$. For example, using a common relay to forward the messages received from all users simultaneously is widely proposed. Under this type of multi-user cooperative transmission protocol, the common relay combines all the relayed packets from each user into one packet (usually using an XOR operation) [7]-[11]. For example, a two user network with this protocol transmits one packet from each user over three time slots instead of the four time slots required using a conventional cooperative protocol. Because the relay needs to combine the users' reencoded packets, DF is used in most such schemes [7]-[10]. In addition, in order to avoid error propagation, the relay checks whether or not all the decoded packets are correct. It drops incorrectly decoded user packets and combines the remaining packets if more than one user's packet is successfully decoded. Therefore, from each user's point of view, selective DF [2] is

\footnotetext{
${ }^{1}$ We consider only communication in which all users are communicating
} with a common destination. employed at the relay node ${ }^{2}$. To the best of our knowledge, in this type of cooperative scheme, the relay is always selective and users' re-encoded data are always XORed e.g. [7]-[10]. The only work using a non-selective protocol, to the best of our knowledge, is [12]. But it is significantly different from the work of this paper. In [12], the relay does not make hard decisions on the users' information bits. Instead, it calculates the log likelihood ratios (LLRs) of the XORed information bit stream based on the soft outputs of the channel decoders (no re-encoding is performed). Then the relay forwards these LLRs. Therefore, the relayed packet is forwarded using analog signals if no quantization is performed. Furthermore, [12] considers only AWGN channels.

Recently, we proposed [1] a DF scheme that forwards hard decisions and reliability information in the form of a quantized SNR value from the relay. A single user network was considered in [1]. It showed that, even though the relay may decode with some errors, the destination can still extract useful information. It suppresses error propagation due to erroneously decoded packets by scaling the soft information for the relay code during decoding at the destination. This allows significant coding gain compared to selective DF.

In this paper, we propose a cooperative protocol for two users using superposition modulation rather than an XOR operation. Here, the data streams from both users are always decoded, interleaved and re-encoded at the common relay (non-selective DF) regardless of their correctness. A recursive systematic convolutional (RSC) code is used by each user and the relay always makes hard decisions, which in principle, requires only Viterbi decoders. The two re-encoded packets are each re-modulated using an $M$-PAM constellation and then one user's $\pi / 2$ rotated symbols are superimposed onto the other user's symbols. This superposition modulation can be considered as re-mapping two users' data onto the inphase and quadrature axes of an $M^{2}$-QAM constellation. The scheme is referred to as superposition mapping (S-mapping) in the sequel. Along with this relayed packet, the average receive SNRs for each user are estimated. The re-mapped packet and receive SNRs are forwarded to the destination. The cooperative

\footnotetext{
${ }^{2}$ Note, due to the existence of multiple users, adaptive DF [2], in which the user sends the relayed code when the relay fails to decode users' information, cannot be used.
} 
Turbo decoder of [1] is used at the destination. Both symmetric and asymmetric network topologies are considered.

As will be discussed in Section IV, we have found that XOR based multi-user cooperative schemes are most suited to those wireless networks in which all users have equal quality channels to both the relay and the destination. This is not the case for a general wireless network where nodes are randomly deployed. In contrast, using s-mapping with a carefully designed distributed error control coding scheme gives better performance even when the users have different channel qualities.

We note that complex field network coding (CFNC) was proposed in [13] using demodulation-and-forward (DemF) and superposition modulation. Although the relay in [13] is also non-selective and uses superposition modulation, there are several key differences between the proposed scheme and the scheme in [13]. In the proposed scheme, full decoding is performed at the relay based on the entire packet instead of symbol by symbol demodulation as in [13]. More importantly, the symbol by symbol demodulation operation at the relay results in a repetition cooperative transmission, while the interleaving and re-encoding used in the proposed scheme results in an incremental cooperative transmission. This allows the formation of a Turbo code for each user. Therefore, the combining scheme of [13] cannot be used here.

This paper is organized as follows. The system model is introduced in Section II. Section III describes the proposed and comparison schemes. Simulation results are presented in Section IV. Finally, conclusions are drawn in Section V.

\section{SYSTEM Description}

The system model considered in this paper is shown in Fig. 1. Two users $\left(U_{1}, U_{2}\right)$ communicate to a common destination $(D)$ aided by one relay $(R)$ node. Each node is assumed to be half duplex and to have only one antenna. The distances between nodes $\left(U_{n}-\mathrm{D}, U_{n}-\mathrm{R}\right.$ and R-D) are normalized against the longest distance, $d_{U_{n} D}^{\max }$, among all the distances so that $d_{U_{n} D}^{\max }=1$. For simplicity, we use a line model, which means the relay is located between the users and the destination and $d_{U_{n} R}+d_{R D}=d_{U_{n} D}$, for $n=1,2$.

Transmission is organized in a packet by packet fashion. The transmission of a packet from each user to the destination is referred to as a transmission cycle. Each cycle is divided into the two stages of broadcast and relay. The broadcast stage is further divided into two time slots. Each user broadcasts its coded message in the assigned time slot during the broadcast stage. The relay decodes and combines through S-mapping both packets into one packet which it forwards to the destination during the relay stage. Therefore, for two users, each transmission cycle consists of three time slots. The channels connecting all nodes are modeled as quasi-static Rayleigh flat fading channels, which are constant over a transmission cycle and change independently between adjacent transmission cycles. The channel coefficient for each channel is modelled as $\rho=\sqrt{g} h$, where $h$ is a circularly symmetric complex Gaussian random variable with zero mean and unit average power and $g$ is the channel gain which is related to distance according to $g=\frac{1}{d^{v}}$, where $v$ is the path-loss exponent.

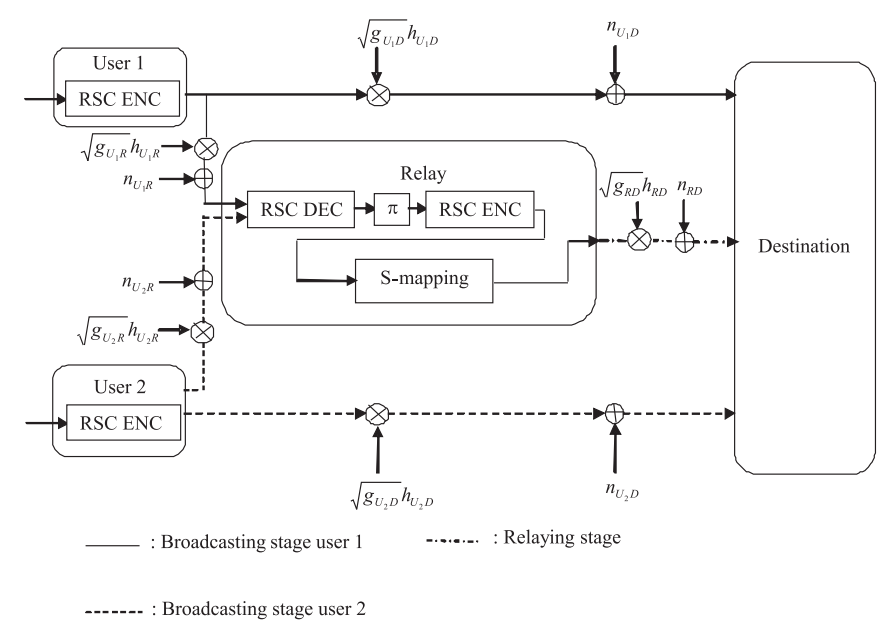

Fig. 1. System model.

During the broadcast stage, each user encodes a block of information bits using a RSC code and then broadcasts this to the destination and relay. The destination delays decoding until the end of the ensuing relay stage. The relay decodes the broadcast messages from both users. Then it interleaves the decoded bits and re-encodes them using the same or a different RSC code. It forwards the combined packet to the destination during the relaying stage (while the source stays silent). The corresponding received signals at the relay and destination for the $m^{t h}$ packet are given by

$$
\begin{aligned}
& \mathbf{y}_{U_{n} R}^{m}=\sqrt{g_{U_{n} R}} h_{U_{n} R}^{m} \mathbf{x}_{U_{n}}^{m}+\mathbf{n}_{U_{n} R}^{m}, \quad n=1,2 \\
& \mathbf{y}_{U_{n} D}^{m}=\sqrt{g_{U_{n} D}} h_{U_{n} D}^{m} \mathbf{x}_{U_{n}}^{m}+\mathbf{n}_{U_{n} D}^{m}, \quad n=1,2 \\
& \mathbf{y}_{R D}^{m}=\sqrt{g_{R D}} h_{R D}^{m} \mathbf{x}_{R}^{m}+\mathbf{n}_{R D}^{m},
\end{aligned}
$$

where $\mathbf{y}_{p q}^{m}$ is the received signal vector at node $q$ sent by node $p, \mathbf{x}_{p}^{m}$ is the encoded symbol vector at node $p$ and $\mathbf{n}_{p q}^{m}$ is the additive white Gaussian noise (AWGN) vector with variance $N_{0} / 2$ per dimension at node $q$ during the transmission from node $p$. Note that, no CRC code is used. So, $\mathbf{x}_{R}^{m}$ may contain errors due to incorrect decoding.

\section{Proposed Two-user Cooperative Protocol and COMPARISON SCHEMES}

\section{A. Proposed Two-user Cooperative Protocol}

In the proposed cooperative scheme, the relay does not check the correctness of the decoded packets. It always decodes the messages from both users, interleaves and reencodes them into two new packets. No CRC code is required, resulting in an always "on" relay for all users. Instead of XORing [14] all user data packets into one packet, we combine them using S-mapping and forward this re-mapped combined packet to the destination. Assuming an $M^{2}$-ary modulation is used at the relay, $\log _{2}(M)$ bits/channel use/user can be achieved during the relay stage, whereas the always "on" DF soft information forwarding schemes of [15], [16] can only achieve $1 \mathrm{bit} / \mathrm{channel}$ use/user. 
Along with the re-encoded relayed packet, the relay forwards the average receive SNR of each user packet,

$$
S N R_{U_{n} R}=\frac{g_{U_{n} R}\left|h_{U_{n} R}\right|^{2} E_{s}}{N_{0}}, n=1,2
$$

where $E_{s}$ is the average symbol energy transmitted from each source to the destination. We call the resulting scheme Smapping with scaling.

Consider the structure of the decoder at the destination. The direct user to destination code is decoded using a standard BCJR algorithm. A modified metric is used for the relayed code. Now we describe how to decode the relayed code for each user. Assuming an $M^{2}$-QAM constellation is used at the relay and the set of constellation points is denoted $\left\{c_{i}\right\}_{i=1}^{M^{2}}$, when the relayed packet is received at the destination, the LLR for the $i^{t h}$ bit of the $k^{t h}$ received symbol is calculated as

$$
\Lambda_{i}^{k}=\log \left(\frac{\sum_{c_{i} \in C^{1}} e^{\left\|y_{R D}^{k}-\rho \cdot c_{i}\right\|^{2} / N_{0}}}{\sum_{c_{i} \in C^{0}} e^{\left\|y_{R D}^{k}-\rho \cdot c_{i}\right\|^{2} / N_{0}}}\right) \quad i=1, \cdots, M^{2}
$$

where $C^{a}$ denotes the set of constellation points having the $i^{\text {th }}$ bit of its binary label equal to $a \in\{0,1\}$. The likelihood for the relayed code of user $\mathrm{n}, \Lambda_{U_{n}}(n \in\{0,1\})$, is obtained by separating these LLR values according to their mapping.

In the cooperative Turbo decoder, the relayed code is decoded using the transition probability for each trellis branch [1],

$$
\gamma_{j}\left(s^{\prime}, s\right)=P\left(u_{j}\right) \exp \left(\frac{-E_{s}}{N_{0}} \cdot \zeta \cdot\|Y-\rho \cdot X\|^{2}\right),
$$

where

$$
\zeta=\frac{\min \left(S N R_{U_{n} R}, S N R_{R D}\right)}{S N R_{R D}}, \quad n=1,2,
$$

$u_{j}$ is the $j^{\text {th }}$ information bit, $P\left(u_{j}\right)$ is its a priori probability, $X$ is the encoder output vector corresponding to the $j^{t h}$ input bit which causes the state change from $s^{\prime}$ to $s, Y$ is the corresponding received signal vector and $\frac{E_{s}}{N_{0}}$ is the average symbol SNR. The component decoder for the code received directly from the user has $\zeta$ set to 1 . The structure of the cooperative Turbo decoder is shown in Fig. 2 and the overall decoder structure at the destination for the S-mapping with the scaling of (6) and (7) is shown in Fig. 3. We assume phase coherence and that any phase ambiguity problem has been resolved. This can be achieved by sending pilot symbols before data transmission, but this is beyond the scope of the current work.

\section{B. Comparative Schemes}

For comparison to the proposed S-mapping with scaling, we consider the following schemes:

(1) Selective XOR: The relay performs a CRC test and relays the data only if it is decoded successfully. If two users' data are decoded successfully, they are reencoded, XORed and forwarded to the destination. Since the relay uses linear codes, the XORed data stream is also a valid codeword [9].

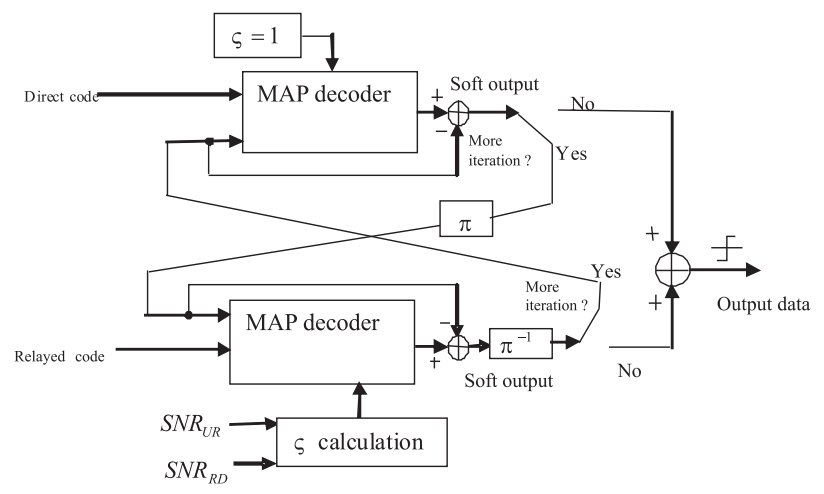

Fig. 2. The structure of the cooperative Turbo decoder.

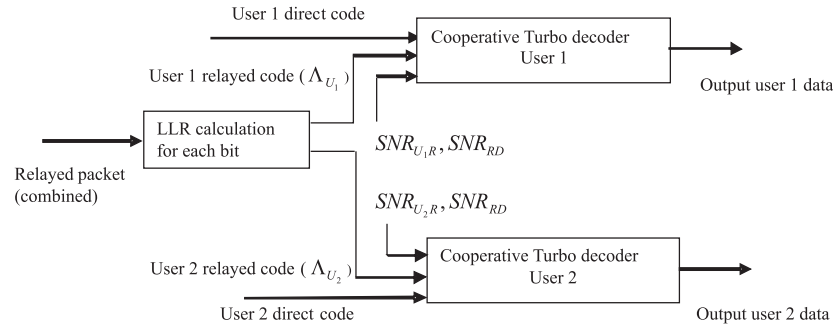

Fig. 3. The decoder structure of the proposed S-mapping based cooperative schemes.

(2) Selective S-mapping: The relay performs a CRC test and only relays the packet if decoded successfully. If both users' data are decoded successfully, they are reencoded, re-mapped onto the constellation points and forwarded to the destination ${ }^{3}$.

(3) Direct transmission: This is not a cooperative scheme. All the coded signals are directly transmitted from the users.

(4) Ideal cooperation: The $U_{n}-\mathrm{R}$ channel is an error free channel.

We do not consider the scheme which non-selectively XORs the data packets as one of the comparative schemes. This is because the equivalent SNR of the packet obtained by using non-selective XOR is much worse than for any of the individual data packets as the errors in each packet are mutually independent.

\section{Simulation Results ANd Discussion}

Our simulations use packets formed by encoding blocks of 500 information bits. We use a path loss exponent $v=3$. The Turbo decoders use 15 iterations. An 8-state rate 1/2 RSC code with generator polynomial $[1,17 / 15]_{8}$ is used at both source and relay. For simplicity, each user and relay use BPSK (effectively 2-PAM) except that QPSK (4-QAM) is used at the relay in the S-mapping based schemes. In order to make a fair comparison, the energy is normalized. The normalization is based on an always "on" relay. The selective schemes will use less power. However, having two users in the system makes

\footnotetext{
${ }^{3}$ Note, all selective schemes need to tell the destination how the relayed packet is formed.
} 


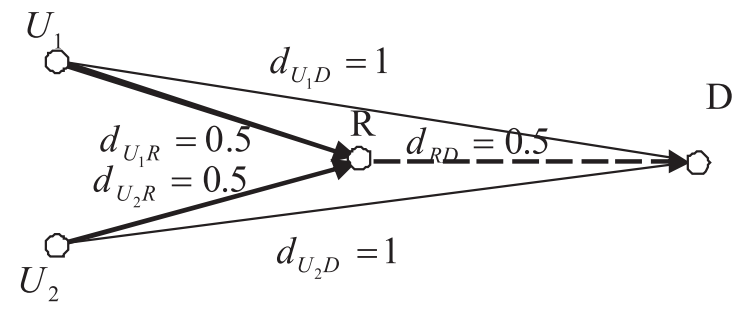

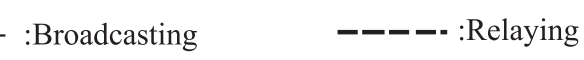

Fig. 4. The symmetric topology.

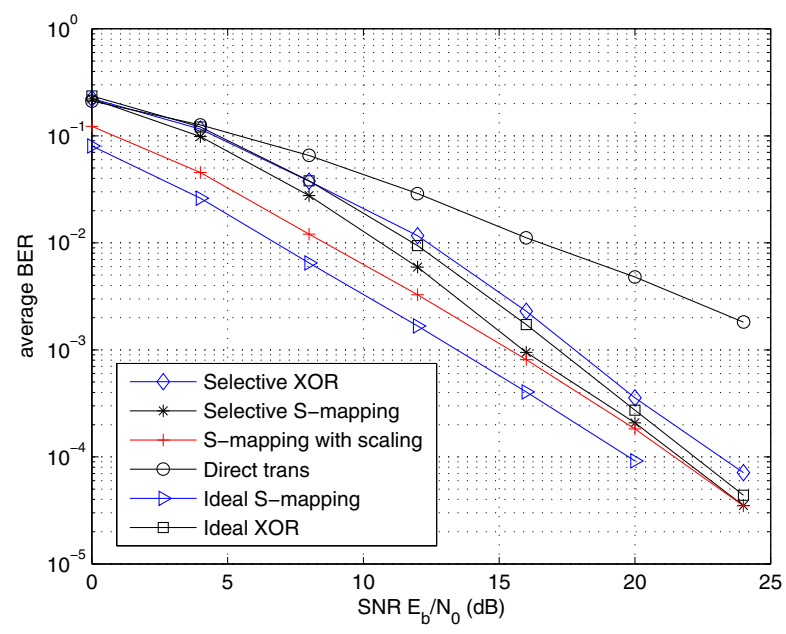

Fig. 5. Average performance for a symmetric network.

this power normalization penalty negligible because the relay is "on" as long as at least one user's information is successfully decoded.

\section{A. Case Study 1: Symmetric Topology}

A symmetric scenario is considered first. This topology is shown in Fig. 4. We assume that the relay is placed at the mid point between users and the destination so that $d_{U_{n} R}=0.5$ for $n=1,2$ and $d_{U_{n} D}=1$ for $n=1,2$.

Fig. 5 shows the resulting average bit error rate (BER) for the various schemes. It clearly shows that, although all schemes achieve a diversity order of 2, S-mapping with scaling achieves the best performance, especially at low SNRs. The selective S-mapping scheme has the same performance as the proposed scheme at high SNRs, while all the XOR schemes perform worse than S-mapping. S-mapping with scaling is within $2 d B$ of the ideal S-mapping scheme over the simulated range of SNR.

Fig. 6 shows the performance of each user under a symmetric network topology. We can see that, since the topology is symmetric, both users yield similar performance.

\section{B. Case Study 2: Asymmetric Topology}

The asymmetric topology of Fig. 7 is now considered. We assume the normalized distances $d_{R D}=0.3, d_{U_{1} R}=0.4$,

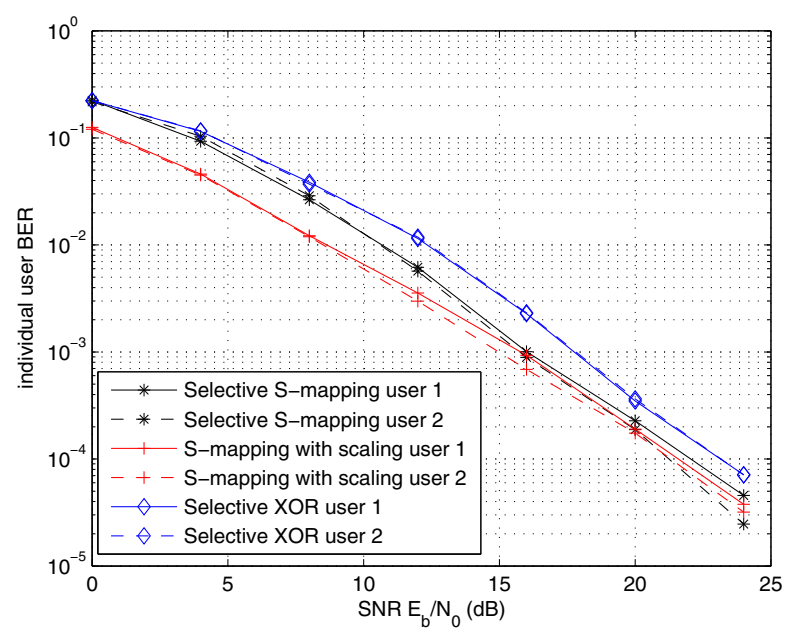

Fig. 6. Performance of individual users for a symmetric network.

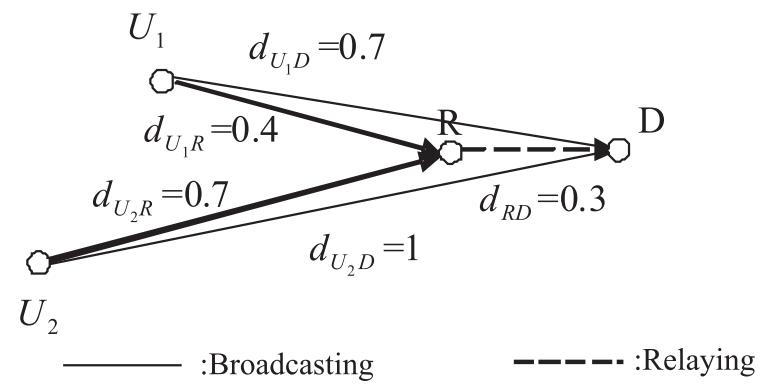

Fig. 7. The considered asymmetric network topology.

$d_{U_{2} R}=0.7, d_{U_{1} D}=0.7$ and $d_{U_{2} D}=1$. The relay is now placed closer to the destination and user 1 has a better average channel condition than user 2 .

Fig. 8 shows the BERs averaged over both users for this case. It clearly shows that the proposed S-mapping with scaling scheme outperforms the other schemes including the ideal XOR scheme over the simulated SNR range, while the selective S-mapping and selective XOR schemes have similar performance.

Fig. 9 shows the performance of each user under the considered asymmetric network topology. We observe that, for user 1, selective S-mapping has better performance than the selective XOR scheme. S-mapping with scaling has better performance than selective S-mapping at low SNR and has similar performance at high SNR. For user 2, both selective schemes have similar performance. S-mapping with scaling improves the performance of user 2 significantly compared to the selective schemes considered here.

The average performances in Figs. 5 and 8 show that using S-mapping with scaling achieves good coding gain compared to both selective schemes under both network topologies. The reason is shown by Fig. 9. It shows that using the selective S-mapping scheme only improves the performance of user 1 (having a better channel condition) compared to the selective XOR scheme while S-mapping with scaling improves the 


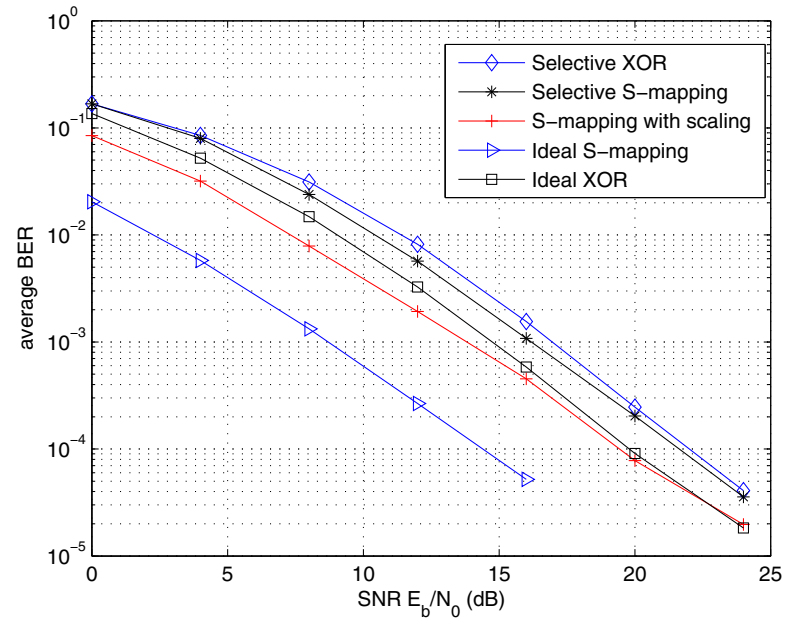

Fig. 8. Average performance for an asymmetric network.

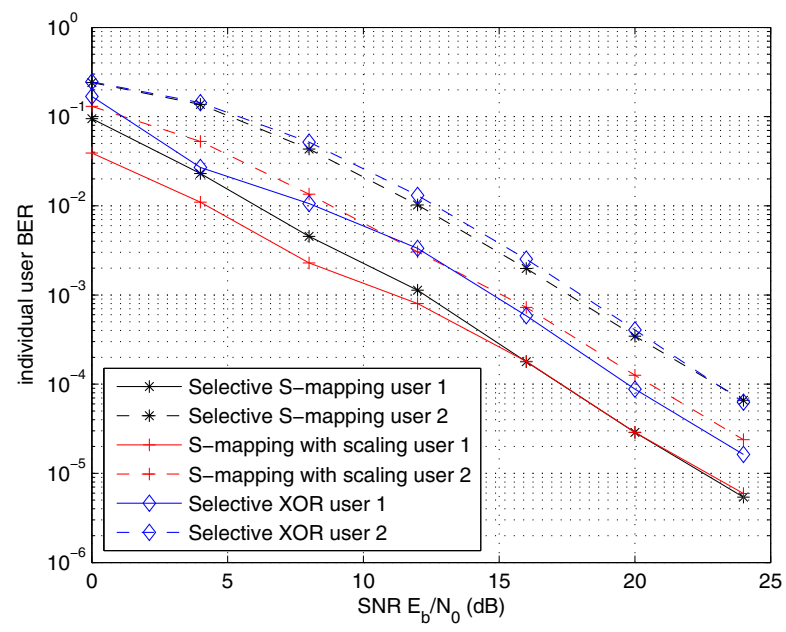

Fig. 9. Performance of individual users for an asymmetric network.

performance of user 2 and maintains the performance of user 1 compared to both selective schemes since it is always "on" for both users. Average performance is dominated by the user with the worst performance.

We have also observed that the S-mapping schemes outperform the XOR based schemes. This problem is caused by the XOR operation used at the relay. Although using XOR enables two users to achieve higher diversity order at an improved rate, it introduces error propagation or correlation between the two users during the decoding process at the destination. This error propagation is more pronounced when the two users have different channel qualities. This degrades the performance of the user having good channel conditions.

\section{CONCLUSiOns AND FurTher WORK}

In this paper, we have proposed a multi-user cooperative scheme using S-mapping and the cooperative Turbo decoder proposed in [1]. It not only achieves good performance in terms of average performance but also reduces the performance gap between different users having different channel conditions without degrading the performance of the user having good channel conditions. The protocol is simple to implement with comparable complexity to other selective schemes in the literature. As a part of ongoing research work, we intend to carry out detailed analysis of the proposed scheme and extend it to larger networks.

\section{REFERENCES}

[1] R. Lin, P. A. Martin, and D. P. Taylor, "Cooperative signalling with soft information combining," Journal of Electrical and Computer Engineering, Hindawi, vol. 2010, Article ID 530190, 2010.

[2] J. N. Laneman, Cooperative diversity in wireless networks: algorithm and architectures. PhD Thesis MIT, 2002.

[3] E. G. Larsson and B. R. Vojcic, "Cooperative transmit diversity based on superposition modulation," IEEE Commun. lett., vol. 9, pp. 778-780, Sep. 2005.

[4] T. Bui and J. H. Yuan, "Iterative approaches of cooperative transmission based on superposition modulation," in Proc. ISCIT, pp. 1423 - 1428, Oct. 17-19 2007.

[5] K. Ishii, "Coded cooperative protocol utilizing superposition modulation for half-duplex scenario," in IEEE Proc. PIMRC, Sep. 3-7 2007.

[6] L. Xiao, T. E. Fuja, J. Kliewer, and J. D. J. Costello, "A network coding approach to cooperative diversity," IEEE Trans. Inform. Theory, vol. 53, pp. 3714-3722, Oct. 2007.

[7] S. Wu, J. Zhu, and M. Zhao, "A novel network-coding-based coded cooperation scheme," in Proc. WCNC, April 2009.

[8] Z. Han, X. Zhang, and H. V. Poor, "High performance cooperative transmission protocols based on multiuser detection and network coding," IEEE Trans. Wireless Commun., vol. 8, pp. 2352-2361, May 2009.

[9] S. Tang, J. Cheng, C. Sun, R. Suzki, and S. Obana, "Turbo network coding for efficient and relaible relay," in Proc. ICCS, Nov. 2008.

[10] X. Bao and J. Li(Tiffany), "Adaptive netwrok coded cooperation ANCC for wireless relay networks: matching code-on-graph with network-ongraph," IEEE Trans. Wireless Commun., vol. 7, pp. 574-583, Feb. 2008.

[11] S. Katti, D. Katabi, W. Hu, H. Rahul, and M. Medard, "The importance of being opportunistic: practical network coding for wireless environments," in Proc. Allerton conference, Sep. 2005.

[12] S. Yang and R. Koetter, "Network coding over a noisy relay: a belief propagation approach," in IEEE Proc. ISIT, 2007.

[13] T. Wang and G. B. Giannakis, "Complex field network coding for multiuser cooperative communication," IEEE J. Select Areas Commun., vol. 26, pp. 561-571, April 2008.

[14] X. Bao and J. Li(Tiffany), "Adaptive network coded cooperation (ANCC) for wireless relay networks: matching code-on-graph with network-on-graph," IEEE Trans. Wireless Commun., vol. 7, pp. 574583, Feb. 2008.

[15] X. Bao and J. Li(Tiffany), "Efficient message relaying for wireless user cooperation: decode-amplify-forward (DAF) and hybrid DAF and coded-cooperation," IEEE Trans. Wireless Commun., vol. 6, pp. 39753984, Nov. 2007.

[16] Y. Li, B. Vucetic, T. F. Wong, and M. Dohler, "Distributed turbo coding with soft information relaying in multihop relay networks," IEEE J. Select Areas Commun., vol. 24, pp. 2040-2050, Nov. 2006. 